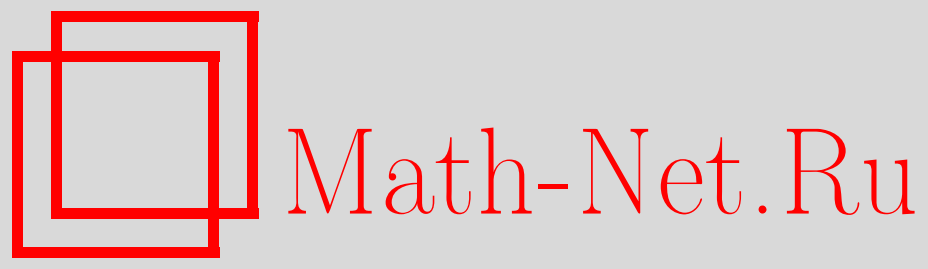

У. Бруццо, Л. Чирио, П. Росси, В. Н. Рубцов, Эквивариантные когомологии и локализация для алгеброидов Ли, Функи. анализ и его прил., 2009, том 43, выпуск 1, 22-36

DOI: https://doi.org/10.4213/faa2940

Использование Общероссийского математического портала Math$\mathrm{Net.Ru}$ подразумевает, что вы прочитали и согласны с пользовательским соглашением http://www. mathnet.ru/rus/agreement

Параметры загрузки:

IP: 18.207 .199 .55

26 апреля 2023 г., 16:53:52

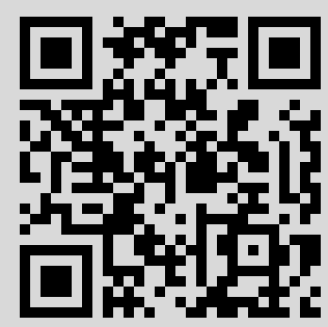


Функционалъный анализ и его приложения

2009, т. 43, вып. 1, с. 22-36

УДК 514.762.32

\title{
Эквивариантные когомологии и локализация для алгеброидов Ли*
}

\author{
(с) 2009. У. Бруццо, Л. Чирио, П. Росси, В. Н. РуБцов
}

Пусть $M$ - многообразие, снабженное действием группы Ли $G$, и $A$ - алгеброид Ли над $M$, снабженный согласованным инфинитезимальным $G$-действием. По этим данным мы строим эквивариантные когомологии алгеброида Ли и доказываем для компактной группы $G$ соответствующую формулу локализации. В качестве приложения мы доказываем аналог формулы Ботта.

\section{§1. Введение}

Понятие алгеброида Ли, которое можно рассматривать как естественное обобщение понятия касательного расслоения к многообразию, позволяет единым образом рассматривать различные геометрические структуры, такие, как пуассоновы многообразия, связности в главных расслоениях, слоения и т. д.

Поскольку довольно часто оказывается возможным связывать алгеброиды Ли и слоения с особенностями, язык алгеброидов Ли может оказаться полезным при обобщении многих конструкций (например, связностей) на особый случай. Недавно было предложено рассматривать новые теоретико-полевые модели, основанные на теории алгеброидов Ли, см., например, [23].

По каждому алгеброиду Ли внутренним образом определяется некоторая теория когомологий. С другой стороны, теория $G$-дифференциальных комплексов, развитая в [12], охватывает случай эквивариантных когомологий алгеброида Ли, которые определены, когда алгеброид Ли допускает (возможно, инфинитезимальное) действие группы Ли $G$, совместимое с ее действием на базовом многообразии $M$.

Возникает естественная проблема - можно ли обобщить на эту ситуацию стандартные формулы локализации для эквивариантных когомологий де Рама. Эта задача представляет также определенный интерес для приложений; так, формулы локализации недавно были использованы при вычислении статистической суммы для $N=2$-суперсимметричной модели Янга-Миллса ([19], [4]). В этом случае соответствующие когомологии представляют собой эквивариантные когомологии де Рама пространства модулей инстантонов - оснащенных самодуальных связностей на $\mathbb{R}^{4}$ (т. е. соответствующий алгеброид Ли является касательным расслоением к пространству модулей инстантонов). Представляется весьма правдоподобным, что аналогичные вычисления для суперсимметричных теорий Янга-Миллса с различным числом суперзарядов могут быть

*Первый и четвертый авторы благодарны Университету Анже и Международной школе высших исследований за гостеприимство и финансовую поддержку во время взаимных визитов в эти институты, а также Федерацию MATPYL. Их исследование выполнено при частичной поддержке ИНФН, проект PI14. 
проведены единым образом при различных выборах структур алгеброидов Ли на пространстве модулей инстантонов.

В этой статье мы рассматриваем формулу локализации для эквивариантных когомологий алгеброида Ли в случае, когда этот алгеброид Ли $A$ над (компактным ориентированным) многообразием $M$ допускает (инфинитезимальное) действие группы Ли. Если «подкрутить» эти когомологии с помощью расслоения ориентации, естественным образом ассоциированного с $A$, то эквивариантный коцикл может быть проинтегрирован по $M$. Если при этом действие группы имеет только изолированные неподвижные точки, то значение этого интеграла может быть выражено с помощью конечной суммы подходящих вычетов в неподвижных точках.

Эта формула локализации сводится, разумеется, к обычной формуле локализации для эквивариантных когомологий де Рама, когда алгеброид Ли $A$ является касательным расслоением $T M$. Подобным же образом она обобщает многие известные формулы локализации, давая их новые доказательства. Так, например, из нее вытекает обобщение на случай алгеброидов Ли разнообразных теорем Ботта о нулях векторных полей (а также соответствующих формул и теоремы Ценкла и Кубарского ([9], [15])). Наша формула обобщает обычную формулу Ботта ровно в том же самом смысле, в котором понятие алгеброида Ли обобщает понятие векторного расслоения. Более того, наша формула локализации применима также в случае, когда $M$ является комплексным многообразием и $A$ есть алгеброид Атьи голоморфного векторного расслоения $E$ над $M$. В этом случае она дает аналог формул локализации Ботта-Баума [1], Черна [10], Kaрелла и Либермана [8], [7] и Лю [16]. Эти аспекты будут рассмотрены в нашей следующей работе [5]. Другая формула локализации для алгеброида Атьи, которую можно получить из нашей, была доказана в [22]. Можно привести еще множество интересных примеров-приложений.

Статья организована следующим образом. В 22 мы приводим базисные определения и некоторые конструкции, относящиеся к теории когомологий алгеброидов Ли. В §3 мы вводим эквивариантные когомологии алгеброидов Ли, следуя, в основном, теории $G$-дифференциалъных комплексов, развитой в [12]. Там же мы доказываем нашу формулу локализации. В §4, следуя [17], мы рассматриваем один из возможных подходов к определению связности в алгеброиде Ли и используем эту связность для построения характеристических эквивариантных классов для алгеброидов Ли. Эти классы используются в $\S 5$ для доказательства аналога формулы Ботта.

Благодарности. Авторы благодарны анонимным рецензентам и члену редколлегии А. Рослому за многочисленные полезные замечания и предложения, которые помогли существенно упростить, сократить и улучшить изложение. Эти предложения позволили нам переосмыслить некоторые принципиальные моменты. Мы благодарны А. Алексееву, П. Бресслеру и И. Косманн-Шварцбах за внимание к работе.

\section{§2. Когомологии алгеброидов Ли}

Пусть $M$ - гладкое многообразие. Будем обозначать через $\mathfrak{X}(M)$ пространство векторных полей на $M$ с естественной скобкой Ли $[\cdot, \cdot]$.

Определение 2.1. Алгеброидом Ли $A$ над $M$ называется векторное расслоение над $M$, снабженное морфизмом векторных расслоений $a: A \rightarrow T M$ 
(называемым якорем) и структурой алгебры Ли на пространстве глобальных сечений $\Gamma(A)$, так что

1) $a: \Gamma(A) \rightarrow \mathfrak{X}(M)$ есть гомоморфизм алгебр Ли;

$2)$ для $\alpha, \beta \in \Gamma(A)$ и для любой функции $f$ выполняется следующее правило Лейбница:

$$
\{\alpha, f \beta\}=f\{\alpha, \beta\}+a(\alpha)(f) \beta
$$

(мы обозначаем через $\{\cdot, \cdot\}$ скобку на $\Gamma(A))$.

Морфизмы между двумя алгеброидами Ли $(A, a)$ и $\left(A^{\prime}, a^{\prime}\right)$ над одним и тем же базовым многообразием $M$ определяются естественным образом, т. е. это морфизмы векторных расслоений $\phi: A \rightarrow A^{\prime}$, такие, что отображение $\phi: \Gamma(A) \rightarrow$ $\Gamma\left(A^{\prime}\right)$ является гомоморфизмом алгебр Ли и очевидная диаграмма, включающая два якоря, коммутативна.

С каждым алгеброидом Ли $A$ можно связать когомологический комплекс $\left(C_{A}^{\bullet}, \delta\right)$, где $C_{A}^{\bullet}=\Gamma\left(\wedge^{\bullet} A^{*}\right)$, а дифференциал $\delta$ определяется формулой

$$
\begin{aligned}
(\delta \xi)\left(\alpha_{1}, \ldots, \alpha_{p+1}\right)= & \sum_{i=1}^{p+1}(-1)^{i-1} a\left(\alpha_{i}\right)\left(\xi\left(\alpha_{1}, \ldots, \hat{\alpha}_{i}, \ldots, \alpha_{p+1}\right)\right) \\
& +\sum_{i<j}(-1)^{i+j} \xi\left(\left\{\alpha_{i}, \alpha_{j}\right\}, \ldots, \hat{\alpha}_{i}, \ldots, \hat{\alpha}_{j}, \ldots, \alpha_{p+1}\right),
\end{aligned}
$$

где $\xi \in C_{A}^{p}$ и $\alpha_{i} \in \Gamma(A), 1 \leqslant i \leqslant p+1$. Получающиеся когомологии $H^{\bullet}(A)$ и называются когомологиями алгеброида Ли $A$.

Замечание 2.2. Следует заметить, что если $A$ есть векторное расслоение, а $\delta$ - дифференцирование степени +1 в градуированной алгебре $\Gamma\left(\wedge^{\bullet} A^{*}\right)$, которое удовлетворяет условию $\delta^{2}=0$, то по $\delta$ можно построить якорь $a: A \rightarrow T M$ и скобку Ли на $\Gamma(A)$, превращающую $A$ в алгеброид Ли. Просто следует положить

$$
\begin{gathered}
a(\alpha)(f)=\delta f(\alpha) \quad \text { для } \alpha \in \Gamma(A), f \in C^{\infty}(M), \\
\xi(\{\alpha, \beta\})=a(\alpha)(\xi(\beta))-a(\beta)(\xi(\alpha))-\delta \xi(\alpha, \beta) \quad \text { для } \alpha, \beta \in \Gamma(A), \xi \in \Gamma\left(A^{*}\right) .
\end{gathered}
$$

Напомним несколько примеров алгеброидов Ли.

Пример 2.3. Инволютивное распределение на касательном расслоении (т. е. слоение) есть алгеброид Ли с инъективным якорем.

Пример $2.4[24]$. Пусть $\mathfrak{M}=(M, \mathscr{F})$ - супермногообразие; в частности, $\mathscr{F}$ есть пучок $\mathbb{Z}_{2}$-градуированных $\mathbb{R}$-алгебр на дифференцируемом многообразии $M$, такой, что его можно реализовать как пучок сечений расслоения алгебры внешних форм $\wedge^{\bullet} E$ векторного расслоения $E$. Пусть $D-$ нечетное векторное поле на $\mathfrak{M}$, такое, что $D^{2}=0$. Тогда $E^{*}$ с якорем и структурой алгебры Ли на $\Gamma\left(E^{*}\right)$, заданными, в соответствии с замечанием 2.2 , полем $D$, которое рассматривается как дифференциал в комплексе $\Gamma\left(\wedge^{\bullet} E\right)$, является алгеброидом Ли. Безусловно, начиная с алгеброида Ли, мы можем построить супермногообразие с нечетным супервекторным полем, квадрат которого равен нулю, так что оба набора данных эквивалентны.

Пример 2.5. Пусть $(M, \Pi)$ - пуассоново многообразие с пуассоновым тензором П. В этом случае $A=T^{*} M$ со скобкой Ли дифференциальных 1-форм

$$
\{\alpha, \beta\}=\mathscr{L}_{\Pi(\alpha)} \beta-\mathscr{L}_{\Pi(\beta)}(\alpha)-d \Pi(\alpha, \beta),
$$


(где $\mathscr{L}$ - производная Ли), а якорь есть пуассонов тензор (точнее, задаваемое им гамильтоново отображение). Когомологии алгеброида Ли $A$ есть когомологии Пуассона-Лихнеровича многообразия $(M, \Pi)$.

Пример 2.6. Пусть $P \stackrel{p}{\rightarrow} M$ - главное расслоение со структурной группой $G$. Тогда имеется так называемая точная последовательность Атьи векторных расслоений над $M$

$$
0 \rightarrow \operatorname{ad}(P) \rightarrow T P / G \rightarrow T M \rightarrow 0
$$

(связность в $P$ есть расщепление этой последовательности). Сечения векторного расслоения $T P / G$ находятся во взаимно однозначном соответствии с $G$-инвариантными векторными полями на $P$. На глобальных сечениях $T P / G$ существует естественная структура алгебры Ли; беря проекцию $T P / G \rightarrow T M$ в качестве якоря, мы получаем алгеброид Ли - алгеброид Amъи, ассоциированный с главным расслоением $P$.

Если $E$ - векторное расслоение над $M$, мы можем также связать с ним алгеброид Атьи: действительно, в этом случае имеется короткая точная последовательность

$$
0 \rightarrow \operatorname{End}(E) \rightarrow \operatorname{Diff}_{0}^{1}(E) \rightarrow T M \rightarrow 0,
$$

где $\operatorname{Diff}_{0}^{1}(E)$ - расслоение дифференциальных операторов 1-го порядка на $E$, имеющих скалярный символ [14], и вновь $\operatorname{Diff}_{0}^{1}(E)$ с естественной структурой алгебры Ли на сечениях и естественной проекцией $\operatorname{Diff}_{0}^{1}(E) \rightarrow T M$ в качестве якоря есть алгеброид Ли. Эти два понятия алгеброида Атьи совпадают, когда $P$ есть расслоение линейных реперов в $E$ (действительно, элемент в $T_{u} P$ задается эндоморфизмом слоя $E_{p(u)}$ и вектором в $\left.T_{p(u)} M\right)$.

Алгеброид Ли с сюръективным якорем (примером которого является алгеброид Атьи) называется транзитивным.

Следуя [11], мы опишем сейчас скрученную форму когомологий алгеброида Ли вместе с естественным спариванием на них. Это будет еще один необходимый ингредиент формулы локализации. Пусть $Q_{A}$ - линейное расслоение $\bigwedge^{r} A \otimes$ $\Omega_{M}^{m}$, где $r=\operatorname{rk} A$ и $m=\operatorname{dim} M$ и $\Omega_{M}^{m}$ есть расслоение дифференциальных $m$-форм на $M$ (далее мы всегда будем обозначать через $\Omega^{m}(M)$ глобальные сечения этого расслоения). Для каждого $s \in \Gamma(A)$ определим отображение $L_{s}=$ $\{s, \cdot\}: \Gamma\left(\wedge^{\bullet} A^{*}\right) \rightarrow \Gamma\left(\wedge^{\bullet} A^{*}\right)$, положив

$$
L_{s}\left(s_{1} \wedge \cdots \wedge s_{k}\right)=\sum_{i=1}^{k} s_{1} \wedge \cdots \wedge\left\{s, s_{i}\right\} \wedge \cdots \wedge s_{k} .
$$

Более того, можно определить отображение

$$
\begin{gathered}
D: \Gamma\left(Q_{A}\right) \rightarrow \Gamma\left(A^{*} \otimes Q_{A}\right)=\Gamma\left(A^{*}\right) \otimes_{C^{\infty}(M)} \Gamma\left(Q_{A}\right), \\
D \tau(s)=L_{s}(X) \otimes \mu+X \otimes \mathscr{L}_{a(s)} \mu,
\end{gathered}
$$

где $\tau=X \otimes \mu \in \Gamma\left(Q_{A}\right)$ и $s \in \Gamma(A)$. Мы рассмотрим скрученный комплекс $\widetilde{C}_{A}^{\bullet}=\Gamma\left(\wedge^{\bullet} A^{*} \otimes Q_{A}\right)=C_{A}^{\bullet} \otimes_{C^{\infty}(M)} \Gamma\left(Q_{A}\right)$, где $\xi \in C_{A}^{\bullet}=\bigoplus_{k=0}^{r} \Gamma\left(\wedge^{k} A^{*}\right)$, с дифференциалом $\tilde{\delta}$, определенным формулой

$$
\tilde{\delta}(\xi \otimes \tau)=\delta \xi \otimes \tau+(-1)^{\operatorname{deg}(\xi)} \xi \otimes D \tau, \quad \xi \in C_{A}^{\bullet} .
$$

Обозначим получившиеся когомологии через $H^{\bullet}\left(A, Q_{A}\right)$. 
Существует естественное отображение ${ }^{1)} p: \widetilde{C}_{A}^{\bullet} \rightarrow \Omega^{\bullet-r+m}(M)$

$$
p(\psi \otimes X \otimes \mu)=(a(\psi\lrcorner X))\lrcorner \mu .
$$

Предложение 2.7. Морфизм р является иепным ${ }^{2)}$ в том смьсле, что диаграмма

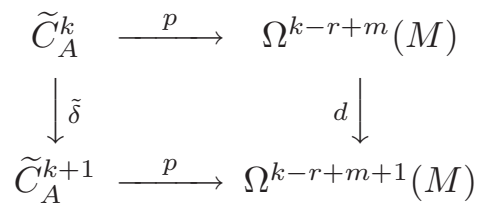

коммутативна с точностью до знака, т.е. на $C_{A}^{k}$

$$
p \circ \tilde{\delta}=(-1)^{k} d \circ p .
$$

Доказательство. Если мы определим производную Ли в $\widetilde{C}_{A}^{\bullet}$, положив $L_{s}=$ $i_{s} \circ \tilde{\delta}+\tilde{\delta} \circ i_{s}$ для элементов $s \in \Gamma(A)$, то получим коммутационное соотношение

$$
p \circ L_{s}=\mathscr{L}_{a(s)} \circ p
$$

на $\widetilde{C}_{A}^{k}$. Используя это тождество, можно доказать (5) с помощью индукции по $k$. Для $k=r$ это тождество сводится к $0=0$. Для малых значений $k$ достаточно проверить тождество для тех $c^{\prime} \in \widetilde{C}_{A}^{k}$, которые могут быть представлены в виде $c^{\prime}=i_{s} c$ для некоторых $s \in \Gamma(A)$ и $c \in \widetilde{C}_{A}^{k+1}$. Результат в этом случае следует из простых вычислений с использованием соотношения (6).

Пусть $M$ - ориентированное компактное многообразие. Заметим, что в силу (канонического) изоморфизма $\widetilde{C}_{A}^{r} \simeq \Omega^{m}(M)$ элементы $\widetilde{C}_{A}^{r}$ можно интегрировать по $M$. Существует невырожденное спаривание

$$
C_{A}^{k} \otimes_{C^{\infty}(M)} \widetilde{C}_{A}^{r-k} \rightarrow \mathbb{R}, \quad \xi \otimes(\psi \otimes X \otimes \mu) \mapsto \int_{M}(\xi \wedge \psi, X) \mu
$$

Для комплекса $\widetilde{C}_{A}^{\bullet}$ имеется версия теоремы Стокса [11]: если $c \in \widetilde{C}_{A}^{r-1}$, то

$$
\int_{M} \tilde{\delta} c=0
$$

Эта формула следует из тождества (5) для $k=r-1$. Отсюда, в свою очередь, вытекает тот факт, что спаривание продолжается в когомологии и определяет билинейное отображение

$$
H^{\bullet}(A) \otimes H^{r-\bullet}\left(A, Q_{A}\right) \rightarrow \mathbb{R} .
$$

Отметим, что это спаривание, вообще говоря, может быть вырожденным.

Мы имеем также естественный морфизм $C_{A}^{\bullet} \otimes_{C^{\infty}(M)} \widetilde{C}_{A}^{\bullet} \rightarrow \widetilde{C}_{A}^{\bullet}$, согласованный со степенями. Переходя снова к когомологиям, мы получаем произведение

$$
H^{i}(A) \otimes H^{j}\left(A, Q_{A}\right) \rightarrow H^{i+j}\left(A, Q_{A}\right) .
$$

1) Мы попеременно используем обозначения $\psi\lrcorner$ и $i_{\psi}$ для свертки (внутреннего произведения) с элементом $\psi$, следуя подходящей формульной эстетике.

2) Мы благодарны А. Алексееву и А. Рослому, указавшим этот факт и предложившим его доказательство. 


\section{§3. Эквивариантные когомологии и локализация}

В этом параграфе мы вводим эквивариантные когомологии для алгеброидов Ли, следуя, в основном, определениям и понятиям, которые использовались в [12] для определения эквивариантных когомологий пуассоновых многообразий (и оставаясь в рамках общей теории эквивариантных когомологий $G$-дифференциальных комплексов, развитой там). Затем мы предлагаем формулу локализации для эквивариантных когомологий алгеброидов Ли (теорема 3.2).

Предположим, что на алгеброиде Ли $A$ задано действие алгебры Ли g, т. е. что существует отображение

$$
b: \mathfrak{g} \rightarrow \Gamma(A) .
$$

Композиция с отображением якоря приводит к гомоморфизму алгебр Ли $\tilde{\rho}=$ $a \circ b: \mathfrak{g} \rightarrow \mathfrak{X}(M)$, т. е. к действию $\mathfrak{g}$ на $M$. Отображения алгебр Ли, подобные $b$, рассматривались в [18] в случае алгеброидов Атьи под названием «представление в дифференцированиях».

Пример 3.1 (см. [25]). Пусть П - регулярный пуассонов тензор на $M$ (т. е. П имеет постоянный ранг), и пусть $\mathscr{S}=\operatorname{Im}(\Pi)$ обозначает соответствующее симплектическое слоение. Семейство симплектических форм, определенных на листах слоения $\mathscr{S}$, задает изоморфизм $\mathscr{S} \simeq \mathscr{S}^{*}$. Следовательно, $\mathscr{S}^{*}$ является подрасслоением в $T M$ и $\Gamma(\operatorname{ker} \Pi)$ есть идеал в $\Omega^{1}(M)$ относительно структуры алгеброида Ли $T^{*} M$, заданной скобкой (1). Более того, $\mathscr{S}^{*}$ является подалгеброидом Ли в $T M$; его когомологии называются касательными когомологиями Лихнеровича-Пуассона. Теперь предположим, что на $M$ задано действие $\rho$ группы Ли $G$; если $\mathfrak{g}$ - алгебра Ли группы $G$, то для каждого $\xi \in \mathfrak{g}$ будем обозначать через

$$
\xi^{*}=\left.\frac{d}{d t} \rho_{\exp (-t \xi)}\right|_{t=0}
$$

соответствующее фундаментальное векторное поле (таким образом, мы получаем гомоморфизм алгебр Ли $\left.\tilde{\rho}: \mathfrak{g} \rightarrow \mathfrak{X}(M), \tilde{\rho}(\xi)=\xi^{*}\right)$. Если $G$-действие является касательным к $\mathscr{S}^{*}$, то мы получим действие $b: \mathfrak{g} \rightarrow \Gamma\left(\mathscr{S}^{*}\right)$ (эта конструкция в [12] называется кокасательным поднятием).

Если для $\xi \in \mathfrak{g}$ точка $x \in M$ является нулем векторного поля $\xi^{*}$, то мы получаем обычный эндоморфизм

$$
L_{\xi, x}: T_{p} M \rightarrow T_{p} M, \quad L_{\xi, x}(v)=\left[\xi^{*}, v\right] .
$$

Рассмотрим градуированное векторное пространство

$$
\mathfrak{A}^{\bullet}=\operatorname{Sym}^{\bullet}\left(\mathfrak{g}^{*}\right) \otimes \Gamma\left(\wedge^{\bullet} A^{*}\right)
$$

с градуировкой

$$
\operatorname{deg}(\mathscr{P} \otimes \beta)=2 \operatorname{deg}(\mathscr{P})+\operatorname{deg} \beta,
$$

где $\mathscr{P} \in \operatorname{Sym}^{\bullet}\left(\mathfrak{g}^{*}\right)$ и $\beta \in \Gamma\left(\wedge^{\bullet} A^{*}\right)$.

Мы будем рассматривать $\mathscr{P}$ как полиномиальную функцию на $\mathfrak{g}$ и определим эквивариантный дифференциал $\delta_{\mathfrak{g}}: \mathfrak{A}^{\bullet} \rightarrow \mathfrak{A}^{\bullet+1}$,

$$
\left(\delta_{\mathfrak{g}}(\mathscr{P} \otimes \beta)\right)(\xi)=\mathscr{P}(\xi)\left(\delta(\beta)-i_{b(\xi)} \beta\right),
$$

где обе части вычисляются на элементе $\xi \in \mathfrak{g}$. Если мы положим $\mathfrak{A}_{G}^{\bullet}=\operatorname{ker} \delta_{\mathfrak{g}}^{2}$, то $\left(\mathfrak{A}_{G}^{\bullet}, \delta_{\mathfrak{g}}\right)$ будет комплексом, когомологии которого мы обозначим через $H_{G}^{\bullet}(A)$ и 
назовем эквивариантными когомологиями алгеброида Ли $A$ (или, более точно, пары $(A, b))$.

Рассматривая градуированное векторное пространство

$$
\mathfrak{Q}^{\bullet}=\mathfrak{A}^{\bullet} \otimes \Gamma\left(Q_{A}\right)=\operatorname{Sym}^{\bullet}\left(\mathfrak{g}^{*}\right) \otimes \Gamma\left(\wedge^{\bullet} A^{*} \otimes Q_{A}\right)
$$

с дифференциалом $\tilde{\delta}_{\mathfrak{g}}$, полученным с помощью дифференциалов $\delta_{\mathfrak{g}}$ и $D$, и полагая $\mathfrak{Q}_{G}^{\bullet}=\operatorname{ker} \tilde{\delta}_{\mathfrak{g}}^{2}$, можно также определить эквивариантные когомологии $H_{G}^{\bullet}\left(Q_{A}\right)$, в которых существует произведение

$$
H_{G}^{i}(A) \otimes H_{G}^{k}\left(Q_{A}\right) \rightarrow H_{G}^{i+k}\left(Q_{A}\right) .
$$

Выпишем теперь формулу локализации. В силу предложения 2.7 ее правая часть может быть вычислена с помощью обычной формулы локализации в эквивариантных когомологиях де Рама; интеграл от эквивариантно замкнутого $\mathfrak{Q}_{G}^{\bullet}$-коцикла $\gamma(\xi)$ является на самом деле интегралом от дифференциальной формы $p(\gamma(\xi))$, и $p(\gamma)$ является коциклом в эквивариантном комплексе де Рама согласно предложению 2.7. Действительно, теперь совсем просто доказать тождество

$$
p\left(\tilde{\delta}_{\mathfrak{g}}(\gamma)\right)=(-1)^{k} d_{\mathfrak{g}}(p(\gamma)),
$$

где $\gamma \in \mathfrak{Q}_{G}^{k}$ (здесь $d_{\mathfrak{g}}-$ дифференциал в обычных эквивариантных когомологиях де Рама). Оно следует из предложения 2.7 и равенств

$$
\begin{aligned}
i_{\xi^{*}} p(\gamma) & \left.\left.\left.\left.\left.\left.=i_{\xi^{*}}[a(\psi\lrcorner X)\right\lrcorner \mu\right]=\left(\xi^{*} \wedge a(\psi\lrcorner X\right)\right)\right\lrcorner \mu=a(b(\xi) \wedge(\psi\lrcorner X)\right)\right\lrcorner \mu \\
& \left.\left.=(-1)^{k-1} a\left(\left(i_{b(\xi)} \psi\right)\right\lrcorner X\right)\right\lrcorner \mu=(-1)^{k-1} p\left(i_{b(\xi)} \gamma\right),
\end{aligned}
$$

если положить $\gamma=\psi \otimes X \otimes \mu$.

Пусть $M$ - замкнутое многообразие с действием $\rho$ компактной группы Ли $G$. Мы предположим также, что $M$ ориентируемо и что $\xi \in \mathfrak{g}$ был выбран так, что $\xi^{*}=\tilde{\rho}(\xi)$ (соответствующие определения см. в примере 3.1 ) имеет только изолированные нули. Обозначим множество таких нулей через $M_{\xi}$. Заметим, что благодаря компактности группы $G$ детерминант $\operatorname{det}\left(L_{\xi, x}\right)$ отличен от 0 в каждом изолированном нуле $x$ и $m$ (размерность $M$ ) обязательно четна (что мы и будем предполагать в дальнейшем).

Если ранг $r$ расслоения $A$ меньше, чем размерность $m$ многообразия $M$, то для каждого эквивариантно замкнутого элемента $\gamma \in \mathfrak{Q}_{G}^{\bullet}$ интеграл $\int_{M} \gamma(\xi)$ равен 0 по соображениям размерности, так как в этом случае $p(\gamma)_{0}=0$. (Здесь индекс 0 обозначает «кусочек» степени 0 в обычной градуировке де Рама.) Поэтому мы будем полагать далее $r \geqslant m$.

Теорема 3.2. Пусть $M$ - замкнутое ориентированное $m$-мерное многообразие, на котором действует компактная группа Ли $G$. Пусть $A-$-лгеброид Ли ранга $r$ над $M$, такой, что $r \geqslant m$, и предположим, что существует гомоморфизм алгебр Ли, такой, что диаграмма

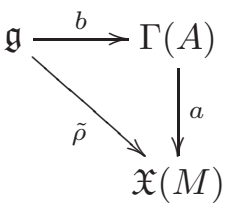


коммутативна; иными словами, отображение г̃ является гомоморфизмом алгебр Ли, $\tilde{\rho}(\xi)=\xi^{*}($ здесъ $\mathfrak{g}-$ алгебра Ли группъ $G)$. Далее предположим, что $\xi \in \mathfrak{g}$ и соответствующее фундаментальное векторное поле $\xi^{*}$ имеет только изолированные нули. Наконец, пусть $\gamma \in \mathfrak{Q}_{G}^{\bullet}-$ эквивариантно замкнутый коиикл, $\tilde{\delta}_{\mathfrak{g}} \gamma=0$.

Тогда имеет место следующая формула локализации:

$$
\int_{M} \gamma(\xi)=(-2 \pi)^{m / 2} \sum_{x \in M_{\xi}} \frac{p(\gamma(\xi))_{0}(x)}{\operatorname{det}^{1 / 2} L_{\xi, x}}
$$

Доказательство. Так как в левой части мы интегрируем обычную дифференциальную форму $p(\gamma(\xi))$, наша формула следует из тождества (11) и обычной формулы локализации.

Легко проверить, что в случае «тривиального» алгеброида, задаваемого на касательном расслоении с тождественным якорем, наша формула сводится к обычной формуле локализации для эквивариантных когомологий де Рама (см., например, [2]).

Замечание 3.3. Если $r \geqslant m$ и ранг линейного отображения $a$ в точке $p$ не максимален (т. е. меньше, чем $m$ ), то $p(\gamma(\xi))_{0}(x)=0$.

Замечание 3.4. В качестве частного случая теоремы 3.2 рассмотрим формулу локализации, связанную с действием векторного поля на $M$. Пусть $M-$ компактное ориентирумое $m$-мерное многообразие, и пусть $X \in \Gamma(T M)-$ векторное поле на $M$ с изолированными нулями, порождающее действие окружности. Пусть $A$ - алгеброид Ли ранга $r$ над $M$, такой, что существует $\widetilde{X} \in \Gamma(A)$, для которого $a(\tilde{X})=X$ (где $a$ - отображение якоря). Тогда для интегрирования формы $\gamma \in \Gamma\left(\wedge^{\bullet} A^{*} \otimes \bigwedge^{r} A \otimes \bigwedge^{m} T^{*} M\right)$, такой, что $\tilde{\delta}_{X} \gamma:=\left(\tilde{\delta}-i_{\tilde{X}}\right) \gamma=0$, применима формула локализации (3.2).

Предположение, что $X$ порождает действие окружности, можно заменить предположением, что $X$ есть изометрия римановой метрики на $M$.

\section{§4. Связности и характеристические классы алгеброидов Ли}

Используя понятие характеристических классов алгеброидов Ли, можно рассмотреть некоторые приложения формул локализации. Мы начнем с введения понятия $A$-связности, см. [17].

Пусть $A$ - алгеброид Ли с якорем $a$, и пусть $P \stackrel{p}{\rightarrow} M-$ главное расслоение со структурной группой $K$. Заметим, что поднятие $p^{*} A=A \times_{M} P$ допускает естественное $K$-действие и $A \simeq p^{*} A / K$. Касательное расслоение $T P$ также допускает естественное $K$-действие, и мы имеем $p_{*}(v k)=p_{*}(v)$ для $v \in T P$ и $k \in K$, и, следовательно, мы имеем индуцированное отображение $p_{*}: T P / K \rightarrow$ $T M$, являющееся якорем алгеброида Атьи, ассоциированного с расслоением $P$, см. последовательность (2).

Определение 4.1. $A$-связностью в $P$ называется отображение расслоений $\eta: p^{*} A \rightarrow T P$, такое, что 
(1) следующая диаграмма коммутативна:

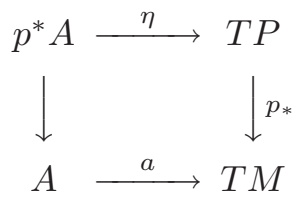

(2) $\eta$ является $K$-эквивариантным, т. е. $\eta(u k, \alpha)=R_{k *} \eta(u, \alpha)$ для всех $k \in K$, $u \in P, \alpha \in A$. (Здесь $R_{k}$ обозначает правое структурное действие элемента $k \in K$ на $P$.)

Если $P$ является расслоением реперов алгеброида Ли $A$, то $\eta$ называется $A$-линейной связностъю.

Будучи эквивариантным отображением, $A$-связность $\eta$ в главном $K$-расслоении определяет отображение расслоений $\omega_{\eta}: A \rightarrow T P / K$, называемое 1 -сечением связности $\eta$. Имеем $p_{*} \circ \omega_{\eta}=a$.

Замечание 4.2. 1. Обычное понятие связности получается, когда мы полагаем $A=T M$, и $\eta$ тогда оказывается соответствующим горизонтальным поднятием, $\eta: p^{*} T M \rightarrow T P$.

2. Обычная связность в $P$ (рассматриваемая как соответствующее горизонтальное поднятие $\zeta: p^{*} T M \rightarrow T P$ ) определяет $A$-связность $\eta$ в $P$, если положить $\eta=\zeta \circ p^{*} a$.

Если $E \stackrel{p_{E}}{\longrightarrow} M$ есть векторное расслоение, ассоциированное с $P$ с помощью представления группы $K$ в некотором векторном пространстве, то $A$-связность в $P$ определяет аналогичную структуру в $E$, т. е. отображение расслоений $\eta_{E}: p_{E}^{*} A \rightarrow T E$, так что диаграмма

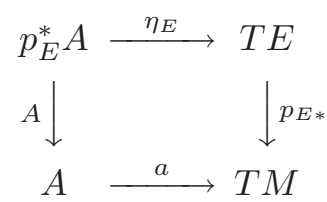

коммутативна. $A$-связность $\eta_{E}$ определяет обычным путем ковариантную производную $\nabla: \Gamma(A) \otimes_{\mathbb{R}} \Gamma(E) \rightarrow \Gamma(E):$ если $\phi: T P / K \rightarrow \operatorname{Diff}_{0}^{1}(E)$ - естественное отображение ${ }^{1)}$, то положим $\nabla_{\alpha}=\left(\phi \circ \omega_{\eta}\right)(\alpha)$. Эта ковариантная производная удовлетворяет правилу Лейбница

$$
\nabla_{\alpha}(f s)=f \nabla_{\alpha}(s)+a(\alpha)(f) s
$$

для любой функции $f$ на $M$.

Введем понятие $G$-эквивариантной $A$-связности. Предположим, что группа Ли $G$ действует на $M$ и что это действие $\rho$ поднимается до действия $\hat{\rho}$ на $A$;

1) Сечение $X$ расслоения $T P / K$ является $K$-инвариантным векторным полем на $P$. Так как имеется очевидное отображение $T P \rightarrow \operatorname{Diff}_{0}^{1}(P \times V)$, где $V$ обозначает стандартный слой расслоения $E$, в силу эквивариантности $X$ задает дифференциальный оператор на $E$ со скалярным символом. 
это означает, что для каждого $g \in G$ мы имеем коммутативную диаграмму

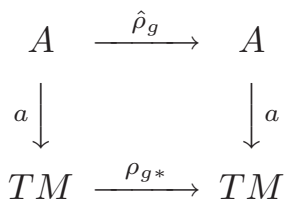

Кроме того, мы предположим, что $\rho$ поднимается также до действия $\tilde{\rho}$ на главном $K$-расслоении $P$, перестановочного со структурным $K$-действием.

Определение 4.3. $G$-эквивариантная $A$-связность $\eta$ в $P$ есть такая $A$-связность $\eta$, что диаграмма

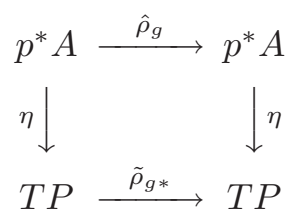

коммутативна для всех $g \in G$.

Так как действие группы $G$ на $P$ перестановочно с действием группы $K$, мы получаем индуцированное действие $\tilde{\rho}_{*}$ на $T P / K$, и утверждение о $G$-эквивариантности связности $\eta$ может быть сформулировано в терминах 1-сечения связности $\omega_{\eta}$ как коммутативность диаграммы

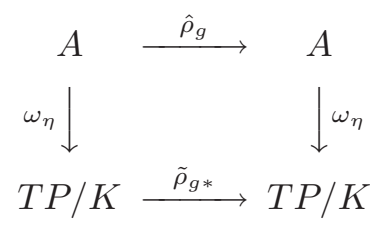

Кривизна $\mathscr{R}_{\eta} A$-связности $\eta$ в главном $K$-расслоении $P$ определяется в терминах отображения $\omega_{\eta}$ как элемент из $\Gamma\left(\wedge^{2} A^{*} \otimes T P / K\right)$, заданный формулой

$$
\mathscr{R}_{\eta}(\alpha, \beta)=\left[\omega_{\eta}(\alpha), \omega_{\eta}(\beta)\right]-\omega_{\eta}(\{\alpha, \beta\}) .
$$

По построению $p_{*} \circ \mathscr{R}_{\eta}=0$, так что $\mathscr{R}_{\eta}$ есть элемент из $\Gamma\left(\Lambda^{2} A^{*} \otimes \operatorname{ad}(P)\right)$. Кривизна $\mathscr{R}_{\eta}$ удовлетворяет аналогу структурных уравнений и тождествам Бъянки. Эти уравнения подходящим образом формулируются в терминах так называемой внешней $A$-производной

$$
\begin{gathered}
D_{A}: \Gamma\left(\wedge^{\bullet} A^{*} \otimes T P / K\right) \rightarrow \Gamma\left(\wedge^{\bullet+1} A^{*} \otimes T P / K\right) \\
\left(D_{A} \chi\right)\left(\alpha_{1}, \ldots, \alpha_{p+1}\right)=\sum_{i=1}^{p+1}(-1)^{i-1}\left[\omega_{\eta}\left(\alpha_{i}\right), \chi\left(\alpha_{1} \ldots, \hat{\alpha}_{i}, \ldots, \alpha_{p+1}\right)\right] \\
\quad+\sum_{i<j}(-1)^{i+j} \chi\left(\left\{\alpha_{i}, \alpha_{j}\right\}, \ldots, \hat{\alpha}_{i}, \ldots, \hat{\alpha}_{j}, \ldots, \alpha_{p+1}\right),
\end{gathered}
$$

в виде

$$
\mathscr{R}_{\eta}=D_{A} \omega_{\eta}-\frac{1}{2}\left[\omega_{\eta}, \omega_{\eta}\right], \quad D_{A} \mathscr{R}_{\eta}=0 .
$$

Заметим, что эквивариантность связности выражается условием

$$
\left[D_{A}, \mathscr{L}_{\tilde{\xi}^{*}}\right]=0
$$


для всех $\xi \in \mathfrak{g}$. Здесь $\mathscr{L}_{\tilde{\xi}^{*}}-$ производная Ли сечений расслоения $T P / K$ относительно сечения $\tilde{\xi}^{*}$ расслоения $T P / K$, индуцированного векторным полем на $P$, порождающим действие группы $G$ (т. е. $\left.\mathscr{L}_{\tilde{\xi}}(v)=\left[\tilde{\xi}^{*}, v\right]\right)$.

Если связность $\eta$ эквивариантна, то мы можем рассматривать эквивариантную версию этих соотношений, определив, в частности, эквивариантную внешнюю $A$-производную $D_{A}^{\mathfrak{g}}$, действующую на $\operatorname{Sym}^{\bullet}\left(\mathfrak{g}^{*}\right) \otimes \Gamma\left(\wedge^{\bullet} A^{*} \otimes T P / K\right)$, формулой

$$
\left(D_{A}^{\mathfrak{g}} \chi\right)(\xi)=D_{A}(\chi(\xi))-\left(i_{\tilde{\xi}^{*}} \otimes \mathrm{id}\right)(\chi(\xi)) .
$$

Кроме того, мы определим эквивариантную кривизну $R_{\eta}^{\mathfrak{g}}$ связности $\eta$ формулой

$$
\left(R_{\eta}^{\mathfrak{g}} \chi\right)(\xi)=R_{\eta}(\chi(\xi))+\mathscr{L}_{\tilde{\xi}^{*}}(\chi(\xi))-\left[D_{A}, i_{\tilde{\xi}^{*}} \otimes \mathrm{id}\right](\chi(\xi))=R_{\eta}(\chi(\xi))+\mu(\chi(\xi)),
$$

где последнее равенство определяет «отображение момента» $\mu$. Далее, квадратные скобки в этих уравнениях являются антикоммутаторами. Легкое вычисление показывает, что эквивариантная кривизна удовлетворяет эквивариантному тождеству Бьянки

$$
D_{A}^{\mathfrak{g}} R_{\eta}^{\mathfrak{g}}=0 .
$$

Мы можем также выразить тождества (15) и (16) в локальной форме, включающей локальное 1-сечение связности, определенное следующим образом. Пусть $\left\{U_{i}\right\}$ - открытое покрытие многообразия $M$, над которым наше расслоение $P$ тривиализуется. Тогда мы получаем локальный изоморфизм

$$
\psi_{j}:\left.(T P / K)\right|_{U_{j}} \rightarrow T U_{j} \times \mathfrak{k},
$$

где $\mathfrak{k}$ - алгебра Ли группы $K$. Локальные 1 -сечения связности определяются условием

$$
\omega_{j}(\alpha)=\operatorname{pr}_{2} \circ \psi_{j} \circ \omega_{\eta}(\alpha)
$$

(где $\mathrm{pr}_{2}$ - проекция на второй сомножитель в $\left.T U_{j} \times \mathfrak{k}\right)$, и по аналогии определим локальные 2-сечения кривизны $\mathscr{R}_{j}$. Тождества (15) теперь запишутся в виде

$$
\mathscr{R}_{j}=\delta \omega_{j}+\frac{1}{2}\left[\omega_{j}, \omega_{j}\right], \quad \delta \mathscr{R}_{j}+\left[\omega_{j}, \mathscr{R}_{j}\right]=0 .
$$

Таким же образом эквивариантная кривизна может быть представлена локальным 2-сечением $\mathscr{R}_{j}^{\mathfrak{g}}$, которое ввиду тождеств (17) удовлетворяет тождествам

$$
\delta_{\mathfrak{g}} \mathscr{R}_{j}^{\mathfrak{g}}+\left[\omega_{j}, \mathscr{R}_{j}^{\mathfrak{g}}\right]=0 .
$$

Гомоморфизм Черна-Вейля определяется следующим образом. Пусть $I^{\bullet}(\mathfrak{k})=$ $\left(\mathrm{Sym}^{\bullet} \mathfrak{k}^{*}\right)^{K}$. Выберем $A$-связность $\eta$ в $P$ и для любого полинома $Q \in I^{\ell}(\mathfrak{k})$ степени $\ell$ определим элемент $\lambda_{Q} \in C_{A}^{2 \ell}$,

$$
\lambda_{Q}\left(\alpha_{1}, \ldots, \alpha_{2 \ell}\right)=\sum_{\sigma}(-1)^{\sigma} \widetilde{Q}\left(\mathscr{R}_{\eta}\left(\alpha_{\sigma_{1}}, \alpha_{\sigma_{2}}\right), \ldots, \mathscr{R}_{\eta}\left(\alpha_{\sigma_{2 \ell-1}}, \alpha_{\sigma_{2 \ell}}\right)\right),
$$

где суммирование ведется по всем перестановкам из $2 \ell$ объектов, а $\widetilde{Q}$ есть поляризация полинома $Q$, т. е. единственная Ad-инвариантная симметрическая функция от $\ell$ переменных в $\mathfrak{k}$, такая, что $\widetilde{Q}(\chi, \ldots, \chi)=Q(\chi)$ для всех $\chi \in \mathfrak{k}$. Можно показать, что это $\delta$-коцикл и что результирующий класс когомологий $\left[\lambda_{Q}\right]$ не зависит от связности и, следовательно, определен гомоморфизм градуированных колец $\lambda: I^{\bullet}(\mathfrak{k}) \rightarrow H^{2 \bullet}(A)$. Если $\tilde{\lambda}: I^{\bullet}(\mathfrak{k}) \rightarrow H_{d R}^{2 \bullet}(M)$ обозначает 
обычный гомоморфизм Черна-Вейля в когомологии де Рама в $M$, то существует коммутативная диаграмма

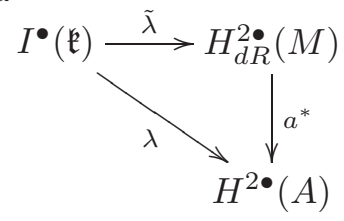

Используя гомоморфизм Черна-Вейля, можно ввести различного сорта характеристические классы для алгеброида Ли $A$. Однако диаграмма (19) показывает, что, к сожалению, эти характеристические классы суть не что иное, как образ при отображении $a^{*}$ характеристических классов расслоения $E$. Чтобы это доказать, выберем любую (обычную) связность в $P$, чтобы вычислить характеристические классы в $H_{d R}^{\bullet}(M)$, и $A$-связность, ассоциированную с ней (см. замечание $4.2(2))$, чтобы вычислить характеристические классы в $H^{\bullet}(A)$. Два таких характеристических класса связаны морфизмом $a^{*}$ из диаграммы (19).

В дальнейшем мы будем работать с характеристическими классами «понтрягинского типа»: а именно, мы возьмем $\mathfrak{k}=\mathrm{gl}(r, \mathbb{C})$, так что $P$ будет расслоением линейных реперов комплексного векторного расслоения $E$. Мы полагаем, что $E$ является комплексификацией некоторого вещественного векторного расслоения. Пусть $Q_{i}$ есть $i$-й элементарный $\mathrm{Ad}$-инвариантный полином, и обозначим через $\lambda_{i}$ соответствующий характеристический класс. Эти характеристические классы равны нулю, когда $i$ нечетно (для проверки зафиксируем связность в $P$, совместимую с послойной метрикой на $E$, и вычислим характеристические классы с помощью индуцированной $A$-связности).

Если $Q \in I^{\bullet}(\operatorname{gl}(r, \mathbb{C}))$ есть $\mathrm{Ad-инвариантный} \mathrm{однородный} \mathrm{симметрический}$ полином степени $2 i$ на алгебре Ли $\operatorname{gl}(r, \mathbb{C})$, то $Q\left(\mathscr{R}_{\eta}^{\mathfrak{g}}\right) \in \mathfrak{A}_{G}^{4 i}$. Легко проверяется следующее утверждение.

Предложение 4.4. Элемент $Q\left(\mathscr{R}_{\eta}^{\mathfrak{g}}\right)$ является $\delta_{\mathfrak{g}}$-замкнутым. Соответствующий класс когомологий $\lambda_{Q}^{\mathfrak{g}}(A) \in H_{G}^{4 i}(A)$ не зависит от эквивариантной связности $\eta$.

Доказательство. Сначала показываем, что элемент $Q\left(\mathscr{R} \frac{\mathfrak{g}}{\eta}\right)$ есть $\delta_{\mathfrak{g}}$-коцикл, используя формулу (18). Чтобы проверить второе утверждение, заметим, что если $\eta, \eta^{\prime}$ - две эквивариантные $A$-связности в главном расслоении $P$, то можно определить 1-параметрическое семейство связностей

$$
\eta_{t}=t \eta^{\prime}+(1-t) \eta
$$

с $0 \leqslant t \leqslant 1$, и пусть

$$
\begin{aligned}
& q\left(\eta, \eta^{\prime}\right)\left(\alpha_{1}, \ldots, \alpha_{2 i}\right) \\
& \quad=i \sum_{\sigma}(-1)^{\sigma} \int_{0}^{1} \widetilde{Q}\left(\frac{d}{d t} \omega_{\eta_{t}}\left(\alpha_{\sigma_{1}}, \alpha_{\sigma_{2}}\right), \mathscr{R}_{\eta_{t}}^{\mathfrak{g}}\left(\alpha_{\sigma_{3}}, \alpha_{\sigma_{4}}\right), \ldots, \mathscr{R}_{\eta_{t}}^{\mathfrak{g}}\left(\alpha_{\sigma_{2 i}-1}, \alpha_{\sigma_{2 i}}\right)\right) d t
\end{aligned}
$$

где $\widetilde{Q}$ - поляризация полинома $Q$. Непосредственное вычисление с использованием тождества (18) и формулы

$$
\frac{d}{d t} \mathscr{R}_{\eta_{t}}^{\mathfrak{g}}=D_{A}^{\mathfrak{g}} \frac{d}{d t} \omega_{\eta_{t}}
$$


показывает теперь, что

$$
Q\left(\mathscr{R}_{\eta^{\prime}}^{\mathfrak{g}}\right)-Q\left(\mathscr{R}_{\eta}^{\mathfrak{g}}\right)=\delta_{\mathfrak{g}} q\left(\eta, \eta^{\prime}\right)
$$

откуда и следует утверждение.

Если $Q$ является стандартным $i$-м элементарным Ad-инвариантным полиномом $\varsigma_{i}$ на $\mathrm{gl}(r, \mathbb{C})$, мы обозначим через $\lambda_{i}^{\mathfrak{g}}(A)(i=1, \ldots, r)$ соответствующий эквивариантный характеристический класс (являющийся нулевым при нечетных $i$ ). Как и выше, эти классы являются просто образами при морфизме $a^{*}$ (сопряженном отображению якоря) эквивариантных классов Черна (комплексифицированного) векторного расслоения $E$.

\section{§5. Формула Ботта}

В качестве приложения нашей теоремы локализации мы докажем результат, который обобщает классическую формулу Ботта [3], а также аналогичные результаты Ценкла и Кубарского [9], [15]. Формула Ботта (в разных вариантах) соответствует разным предположениям относительно векторного поля, входящего в формулу. Рассматриваемый нами случай обобщает обычную формулу Ботта для векторного поля, порождающего действие окружности и имеющего изолированные особенности.

Пусть $\Phi$ есть моном от $n=[r / 4]$ переменных. Обозначим через $W_{\Phi}$ его noлный вес, определенный приписыванием веса $4 i$ его $i$-й переменной. Мы свяжем с мономом $\Phi$ вещественное число, сопоставляемое алгеброиду $A$. Предположим, что $W_{\Phi} \leqslant r$, и пусть $\Xi^{\mathfrak{g}}$ есть эквивариантный скрученный коцикл степени $r-W_{\Phi}$, т. е. $\Xi^{\mathfrak{g}} \in \mathfrak{Q}_{G}^{r-W_{\Phi}}$. Пусть $\Xi=\Xi^{\mathfrak{g}}(0) \in \widetilde{C}_{A}^{r-W_{\Phi}}$ обозначает член степени нуль в $\Xi^{\mathfrak{g}}($ как функции от $\xi \in \mathfrak{g})$. Он удовлетворяет соотношению $\tilde{\delta} \Xi=0$. Можно положить

$$
\Phi_{\Xi}(A)=(-2 \pi)^{-m / 2} \int_{M} \Phi\left(\lambda_{2}(A), \ldots, \lambda_{2 n}(A)\right) \wedge \Xi,
$$

где $\lambda_{i}$ - характеристические классы векторного расслоения $A$ (они были определены в предыдущем параграфе). Это число зависит только от алгеброида Ли $A$ и от класса когомологий $\Xi$. Мы также определим элемент

$$
\begin{aligned}
\Phi_{\Xi}^{\mathfrak{g}}(A) & =(-2 \pi)^{-m / 2} \int_{M} \Phi\left(\lambda_{2}^{\mathfrak{g}}(A), \ldots, \lambda_{2 n}^{\mathfrak{g}}(A)\right) \wedge \Xi^{\mathfrak{g}} \\
& =(-2 \pi)^{-m / 2} \int_{M} \Phi\left(\varsigma_{2}\left(\mathscr{R}_{\eta}+\mu\right), \ldots, \varsigma_{2 n}\left(\mathscr{R}_{\eta}+\mu\right)\right) \wedge \Xi^{\mathfrak{g}}
\end{aligned}
$$

в $\operatorname{Sym}^{\bullet}\left(\mathfrak{g}^{*}\right)$. Разумеется, $\Phi_{\Xi}(A)=\Phi_{\Xi}^{\mathfrak{g}}(A)(0)$. Получаем следующий результат в духе формулы Ботта, который является непосредственным следствием из теоремы 3.2 .

Теорема 5.1. Пусть $A$ - алгеброид Ли ранга $r$ над ориентированным многообразием $M$, и пусть $\alpha \in \Gamma(A)$ - сечение, порожсдающее действие окружности на $A$ и такое, что а $(\alpha)$ имеет изолированные нули. Пусть $\Phi-$ полином от $n=[r / 4]$ переменных, мономы которого имеют полный вес $W_{\Phi}$. Тогда если $r \geqslant m \geqslant W_{\phi}$, mо

$$
\Phi_{\Xi}(A)=\sum_{x \in M_{a(\alpha)}} \frac{\Phi\left(c_{2}\left(L_{a(\alpha), x}\right), \ldots, c_{2 n}\left(L_{a(\alpha), x}\right)\right) p\left(\Xi^{\mathfrak{g}}\right)_{0}}{\operatorname{det}^{1 / 2} L_{a(\alpha), x}}
$$


где классы $c_{i}\left(L_{a(\alpha), x}\right)$ суть эквивариантные классьь Черна эндоморфизма $L_{a(\alpha), x}$, действующего в касательном пространстве $T_{x} M$ в нуле $x$ поля $a(\alpha)$ (cM. [3]).

Доказательство. Правая часть формулы (21) получается из правой части формулы (13), если учесть следующие два факта. Первый: мы можем вычислить эквивариантные характеристические классы, входящие в $\Phi_{\Xi}^{\mathfrak{g}}(A)$, выбирая в главном расслоении $G L(A)$ эквивариантную $A$-связность, индуцированную обычной эквивариантной связностью $\zeta$ в векторном расслоении $A$. Таким образом,

$$
\Phi\left(\lambda_{2}^{\mathfrak{g}}(A), \ldots, \lambda_{2 n}^{\mathfrak{g}}(A)\right)=a^{*}\left(\Phi\left(\nu_{2}^{\mathfrak{g}}(A), \ldots, \nu_{2 n}^{\mathfrak{g}}(A)\right)\right),
$$

где классы $\nu_{i}^{\mathfrak{g}}$ - это эквивариантные классы Черна комплексификации векторного расслоения $A$. Второй: если $R_{\zeta}^{\mathfrak{g}}$ есть эквивариантная кривизна связности $\zeta$, то для каждой элементарной симметрической функции $\varsigma_{i}$ и каждого нуля $x$ поля $a(\alpha)$

$$
\left(\varsigma_{i}\left(R_{\zeta}^{\mathfrak{g}}\right)\right)_{0}(x)=c_{i}\left(L_{a(\alpha), x}\right) .
$$

Если $A=T M$, формула (21) сводится к обычной формуле Ботта.

\section{§6. Заключительные замечания}

Как мы покажем в работе [5], теорема 5.1 обобщает несколько формул локализации для случая действия голоморфного поля на комплексном многообразии, которое эквивариантно поднимается до действия на голоморфном векторном расслоении, см. [1], [10], [8], и, в частности, воспроизводит теорему о вычетах Гротендика.

С другой стороны, наша формула допускает обобщения в нескольких направлениях. Одним из них могла бы быть формула локализации для эквивариантных когомологий, ассоциированных с действием группы Ли на алгеброиде Куранта. Такая формула объединяла бы несколько формул, появившихся недавно в литературе и связанных, в основном, с обобщенными структурами КалабиЯу ([6], [20], [21], [13]), и давала бы нашу формулу в случае замены алгеброида Куранта на алгеброид Ли.

\section{ЛитерАТУРА}

[1] P. F. Baum, R. Bott, On the zeroes of meromorphic vector fields, in: Essays in Topology and Related Topics. Memoires dédiés à Georges de Rham, Springer-Verlag, New York, 1970, 29-47.

[2] N. Berline, E. Getzler, M. Vergne, Heat Kernels and Dirac Operators, Grundlehren der Mathematischen Wissenschaften, Bd. 298, Springer-Verlag, Berlin, 1992.

[3] R. Bott, Vector fields and characteristic numbers, Michigan Math. J., 14 (1967), 231244.

[4] U. Bruzzo, F. Fucito, J. F. Morales, A. Tanzini, Multi-instanton calculus and equivariant cohomology, J. High Energy Physics, 2003:5 (2003), 54-76 (electronic).

[5] U. Bruzzo, V. Rubtsov, Holomorphic Atiyah algebroids and localization (в печати).

[6] H. Bursztyn, G. R. Cavalcanti, M. Gualtieri, Reduction of Courant algebroids and generalized complex structures, Adv. Math., 211:2 (2007), 726-765.

[7] J. B. Carrell, A remark on the Grothendieck residue map, Proc. Amer. Math. Soc., 70:1 (1978), 43-48. 
[8] J. B. Carrell, D. I. Lieberman, Vector fields and Chern numbers, Math. Ann., 225:3 (1977), 263-273.

[9] B. Cenkl, Zeros of vector fields and characteristic numbers, J. Diff. Geom., 8 (1973), $25-46$.

[10] S.-S. Chern, Meromorphic vector fields and characteristic numbers, Scripta Math., 29:3-4 (1973), 243-251.

[11] S. Evens, J.-H. Lu, A. Weinstein, Transverse measures, the modular class and a cohomology pairing for Lie algebroids, Quart. J. Math. Oxford, Ser. 2, 50 (1999), 417-436.

[12] V. L. Ginzburg, Equivariant Poisson cohomology and a spectral sequence associated with a moment map, Internat. J. Math., 10 (1999), 977-1010.

[13] S. Hu, B. Uribe, Extended manifolds and extended equivariant cohomology, http://arxiv.org/abs/math/0608319.

[14] Y. Kosmann-Schwarzbach, Vector fields and generalized vector fields on fibered manifolds, in: Geometry and Differential Geometry (Proc. Conf. Univ. Haifa, Israel, 1979), Lecture Notes in Math., vol. 792, Springer-Verlag, Berlin, 1980, 307-355.

[15] J. Kubarski, Bott's vanishing theorem for regular Lie algebroids, Trans. Amer. Math. Soc., 348:6 (1996), 2151-2167.

[16] K. Liu, Holomorphic equivariant cohomology, Math. Ann., 303:1 (1995), 125-148.

[17] R. Loja Fernandes, Lie algebroids, holonomy and characteristic classes, Adv. Math., 170:1 (2002), 119-179.

[18] K. C. H. Mackenzie, General theory of Lie groupoids and Lie algebroids, London Math. Soc. Lecture Note Series, vol. 213, Cambridge University Press, Cambridge, 2005.

[19] N. A. Nekrasov, Seiberg-Witten potential from instanton counting, Adv. Theor. Math. Phys., 7:5 (2003), 831-864.

[20] Y. Nitta, Reduction of generalized Calabi-Yau structures, J. Math. Soc. Japan, 59:4 (2007), 1179-1198.

[21] Y. Nitta, Duistermaat-Heckman formula for a torus action on a generalized CalabiYau manifold and localization formula, http://arxiv.org/abs/math/0702264v1.

[22] В. Рубцов, Топологические инварианты эллиптических комплексов Спенсера уравнений Ли, Известия ВУЗов, Математика, 1992:5 (1992), 74-92.

[23] T. Strobl, Algebroid Yang-Mills theory, Phys. Rev. Lett., 93:21 (2004), 211601.

[24] А. Ю. Вайнтроб, Алгеброидъ Ли и гомологические векторные поля, УМН, 52:2 (1997), 161-162.

[25] I. Vaisman, Remarks on the Lichnerowicz-Poisson cohomology, Ann. Inst. Fourier (Grenoble), 40:4 (1990), 951-963.

Scuola Internazionale Superiore di Studi Avanzati, Trieste Istituto Nazionale di Fisica Nucleare, Sezione di Trieste e-mail: bruzzo@sissa.it

Scuola Internazionale Superiore di Studi Avanzati, Trieste Istituto Nazionale di Fisica Nucleare, Sezione di Trieste e-mail: cirio@sissa.it

Istituto Nazionale di Fisica Nucleare, Sezione di Trieste e-mail: issoroloap@gmail.com

Université d'Angers, Département de Mathématiques

ИТЭФ, теоретический отдел, Москва

e-mail: volodya@math.cnrs.fr
Поступило в редакцию

17 января 2006 г.

В переработанном виде

28 июня 2008 г. 